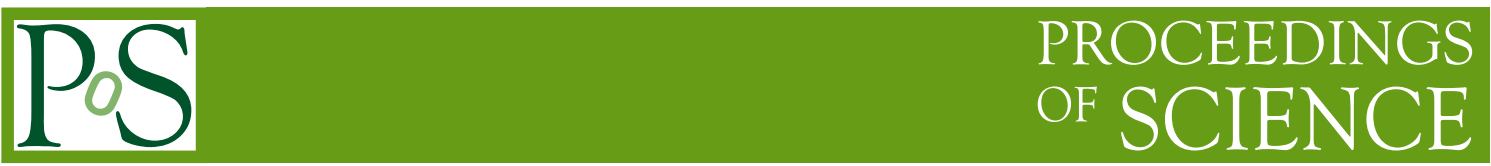

\title{
Search for charged Higgs bosons in ATLAS using other final states
}

\author{
Carlos Sandoval*, on behalf of the ATLAS collaboration \\ Universidad Antonio Nariño \\ E-mail: carlos.sandovalecern.ch
}

Three different searches for charged Higgs bosons with the ATLAS detector are presented. The first one is a search for a charged Higgs boson in $t \bar{t}$ decays, where one of the top quarks decays via $t \rightarrow H^{+} b$, followed by $H^{+} \rightarrow c \bar{s}$ [1]. 95\% confidence level (CL) upper limits on $B\left(t \rightarrow b H^{+}\right)$ varying between $5 \%$ and $1 \%$ for $H^{+}$masses between $90 \mathrm{GeV}$ and $150 \mathrm{GeV}$, assuming $B\left(H^{+} \rightarrow\right.$ $c \bar{s})=100 \%$ are obtained. The second one is a search for charged Higgs bosons through the violation of lepton universality in $t \bar{t}$ events, where signatures containing leptons $(e / \mu)$ and/or a hadronically decaying $\tau\left(\tau_{\text {had }}\right)$ were used [2]. No significant deviation from the Standard Model predictions is observed. With the assumption that the branching fraction $B\left(H^{+} \rightarrow \tau v\right)$ is $100 \%$, upper limits in the range $3.2 \%$ to $4.4 \%$ on the branching fraction $B\left(t \rightarrow b H^{+}\right)$for charged Higgs boson masses in the range $90-140 \mathrm{GeV}$ are obtained. Finally, a search for new particles in an extension to the Standard Model that includes a heavy Higgs boson $\left(H^{0}\right)$, an intermediate charged Higgs-boson pair $\left(H^{ \pm}\right)$, and a light Higgs boson $\left(h^{0}\right)$ [3], is presented. The data is found to be consistent with Standard Model predictions, and 95\% confidence-level upper limits on the product of cross section and branching ratio are obtained. The limits range from 0.065 to $43 \mathrm{pb}$ as a function of $H^{0}$ and $H^{ \pm}$masses, with $m_{h^{0}}$ fixed at $125 \mathrm{GeV}$.

Prospects for Charged Higgs Discovery at Colliders - CHARGED 2014,

16-18 September 2014

Uppsala University, Sweden

\footnotetext{
* Speaker.
} 


\section{Introduction}

Recently, a Higgs boson has been discovered by the ATLAS [4] and CMS [5] Collaborations with a mass of approximately $125 \mathrm{GeV}$. This particle seems to have the characteristics of the standard model (SM) Higgs boson so far. Beyond the SM, many models have been proposed, extending the Higgs sector to explain electroweak symmetry breaking. The newly discovered boson is compatible with many of these models so that discovering its true nature is crucial to understanding EWSB. Two Higgs-doublet models (2HDM) [6] are simple extensions of the SM with five observable Higgs bosons, of which two are charged $\left(H^{+}\right.$and $\left.H^{-}\right)$, and three are neutral $\left(h^{0}, H^{0}\right.$ and $\left.A^{0}\right)$. The discovery of a charged Higgs boson would be a signal for new physics beyond the SM. In several models, e.g. a type-II 2HDM describing the Higgs sector of the Minimal Supersymmetric extension of the Standard Model (MSSM) [7], the main production mode for charged Higgs bosons with a mass $m_{H^{+}}$smaller than the top quark mass $\left(m_{t}\right)$ is through top quark decays $t \rightarrow b H^{+}$. The dominant source of top quarks at the Large Hadron Collider (LHC) is through $t \bar{t}$ production.

At tree level, the MSSM Higgs sector is determined by two independent parameters, which can be taken to be the mass $m_{H^{+}}$and $\tan \beta$. In the MSSM, a light $H^{+}$(defined as $m_{H^{+}}<m_{t}$ ) decays predominantly to $c \bar{s}, \tau v$, and $b \bar{b} W^{+}$, with the respective branching ratios depending on $\tan \beta$ and $m_{H^{+}}$. For $\tan \beta<1, c \bar{s}$ is an important decay mode with $B\left(H^{+} \rightarrow c \bar{s}\right)$ near $70 \%$ [8] for $m_{H^{ \pm}}=110$ $\mathrm{GeV}$, whereas for $\tan \beta>3, H^{+} \rightarrow \tau \nu$ dominates (90\%). For higher $H^{+}$masses at low $\tan \beta$, the decay mode $H^{+} \rightarrow W b \bar{b}$ can be dominant. Three different searches for charged Higgs bosons with the ATLAS detector [9] exploring these scenarios are presented, the first two (the $\mathrm{H}^{+} \rightarrow c \bar{s}$ search [1] and the $H^{+} \rightarrow \tau \nu$-ratio method search [2]), were done using a dataset corresponding to an integrated luminosity of $4.7 \mathrm{fb}^{-1}$ recorded by the ATLAS detector in proton-proton collisions at a centre-of-mass energy of $\sqrt{s}=7 \mathrm{TeV}$. The multi-higgs-boson cascade search [3] uses the dataset corresponding to $20.3 \mathrm{fb}^{-1}$ recorded by the ATLAS detector in proton-proton collisions at a centre-of-mass energy of $\sqrt{s}=8 \mathrm{TeV}$.

\section{2. $H^{+} \rightarrow c \bar{s}$ search}

A set of requirements is imposed to select events containing $t \bar{t}$ decays in the lepton+jets channel. First, events are required to contain a primary vertex with at least five associated tracks to suppress non-collision backgrounds. Exactly one electron with a large transverse energy $\left(E_{\mathrm{T}}>25\right.$ $\mathrm{GeV}$ ) and $|\eta|<2.5$, excluding the barrel-endcap transition region $1.37<|\eta|<1.52$, or one muon with large transverse momentum $\left(p_{\mathrm{T}}>20 \mathrm{GeV}\right)$ and $|\eta|<2.5$ is required. The selected lepton must match a lepton trigger object that caused the event to be recorded. Jets present in $W / Z+$ jet events tend to originate from soft gluon emissions. These backgrounds are therefore reduced by requiring at least four jets with $p_{\mathrm{T}}>25 \mathrm{GeV}$ and $|\eta|<2.5$. At least two jets must be identified as originating from a $b$-decay. To suppress backgrounds from QCD multi-jet events, the missing transverse momentum is required to be $E_{\mathrm{T}}^{\text {miss }}>20(30) \mathrm{GeV}$ in the muon (electron) channel. Further reduction of the multi-jet background is achieved by requiring the transverse mass $\left(m_{\mathrm{T}}\right)$ of the lepton and $E_{\mathrm{T}}^{\text {miss }}$ to satisfy $m_{\mathrm{T}}>30 \mathrm{GeV}$ in the electron channel and $\left(E_{\mathrm{T}}^{\text {miss }}+m_{\mathrm{T}}\right)>60 \mathrm{GeV}$ in the muon channel. In the selected events, the two jets originating from the decay of the $\mathrm{H}^{+}$must be identified in order to reconstruct the mass. A kinematic fitter [10] is used to identify and reconstruct the mass 
of dijets from $W / H^{+}$candidates, by fully reconstructing the $t \bar{t}$ system. In the kinematic fitter, the lepton, $E_{\mathrm{T}}^{\mathrm{miss}}$ (assumed to be from the neutrino), and four jets are assigned to the decay particles from the $t \bar{t}$ system. The longitudinal component of the neutrino momentum is calculated from the constraint that the invariant mass of the leptonic $W$ boson decay products must be the experimental value. The fitter also constrains the invariant mass of the two systems $(b l v, b j j)$ to be within $\Gamma_{t}=1.5 \mathrm{GeV}$ of the top-quark mass $172.5 \mathrm{GeV}$. When assigning jets in the fitter, $b$-tagged jets are assumed to originate from the $b$-quarks. The best $b b j j$ combination is found by minimising a $\chi^{2}$ for each assignment of jets to quarks and for the choice of solution for the longitudinal neutrino momentum, where the five highest- $p_{\mathrm{T}}$ jets are considered as possible top-quark decay products. The combination with the smallest $\chi^{2}$ value, $\chi_{\text {min }}^{2}$, is selected as the best assignment.

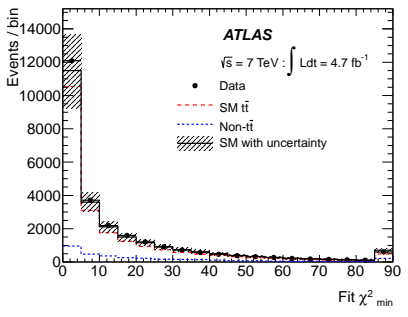

(a)

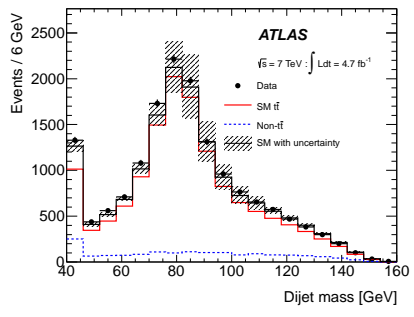

(b)

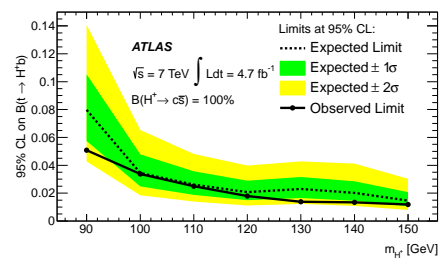

(c)

Figure 1: (a) Comparison of the distribution of $\chi^{2}$ from the kinematic fitter for data and the expectation from the background estimates for the combined electron and muon channels. (b) The dijet mass distribution from data and the expectation from the SM $(B=0)$. (c) The extracted 95\% C.L. upper limits on $B\left(t \rightarrow H^{+} b\right)$ are shown in the range of the charged Higgs mass from 90 to $150 \mathrm{GeV}$. The limits shown are calculated using the CLs limit-setting procedure [1].

The $\chi_{\min }^{2}$ distribution for selected events in the data agrees well with the expectation from the simulation as shown in Figure 1 (a). Events are required to have $\chi_{\min }^{2}<10$ to remove poorly reconstructed $t \bar{t}$ events. This selection has an efficiency of $63 \%$ for SM $t \bar{t}$ events. The fit results in a $12 \mathrm{GeV}$ dijet mass resolution. This is a 20 to $30 \%$ improvement, depending on the mass of the boson studied, compared to the resolution obtained when the same jets are used with their original transverse momentum measurements. After the fit, there is better discrimination between the mass peaks of the $W$ boson from SM decays of $t \bar{t}$ and a $110 \mathrm{GeV} H^{+}$boson in this example.

The background estimates and the estimate of the signal efficiency are subject to a number of systematic uncertainties. The QCD multi-jet background is estimated using a data-driven method [11] that employs a likelihood fit to the $E_{\mathrm{T}}^{\text {miss }}$ distribution in the data, using a template for the multi-jet background and templates from MC simulations for all other processes. The dijet mass distribution of multi-jet events (Figure 1](b)) is obtained from a control region in the data, where leptons are required to be semi-isolated [1].The rate of $W+$ jets events is estimated by a data-driven method [12] that uses the observed difference in the number of $W^{+}$and $W^{-}$bosons in the data and the charge asymmetry $\left(W^{+}-W^{-}\right) /\left(W^{+}+W^{-}\right)$, which is calculated to good precision by the MC simulation of $W+$ jets events. Uncertainties on the modelling of the detector and on theory give rise to systematic uncertainties on the signal and background rate estimates. The effects of the systematic uncertainties are comparable, within $10 \%$, between the SM and signal $t \bar{t}$ samples. 
The combined uncertainty on the single top-quark and diboson backgrounds is $15 \%$, which comes mostly from the uncertainties on the cross-section, jet energy scale, and $b$-tagging. The total uncertainty on the overall normalisation of the non- $t \bar{t}$ backgrounds is $30 \%$.

The limits on the branching ratio are extracted using the confidence level technique (CL) at 95\% [13]. The consistency of the data with the background model can be determined by comparing the value of the test statistic in the data with the expectation from background-only Monte Carlo simulated experiments. The corresponding probability ( $p$-value) for the background to produce the observed mass distribution varies from $67 \%$ to $71 \%$ as a function of $m_{H^{+}}$, indicating that there is no significant deviation from the background hypothesis. The observed limits, including both statistical and systematic uncertainties, vary between $1 \%$ and $5 \%$ (Figure 1(c)). The extracted limits are stringent on the branching ratio $\mathrm{Br}\left(t \rightarrow H^{+} b\right)$, assuming $\mathrm{Br}\left(H^{+} \rightarrow c \bar{s}\right)=100 \%$.

\section{3. $H^{+} \rightarrow \tau \nu$ (ratio method) search}

This search used an alternative technique for $\mathrm{H}^{+}$searches in the mass range $90-160 \mathrm{GeV}$. Instead of using the shape of discriminating variables in order to search for a local excess of events above the predicted SM background, this analysis is based on the measurement of a ratio of event yields between two $t \bar{t}$ final states, which in turn allows for the cancellation of most of the systematic uncertainties. In top quark decays, $W$ bosons decay equally to leptons of the three generations, while $H^{+}$may decay predominantly into $\tau \nu$. Hence, an excess of $t \bar{t}$ events with at least one hadronically decaying $\tau$ lepton ( $\tau_{\text {had }}$ ) in the final state, as compared to the rate for $t \bar{t}$ events with only electrons and/or muons, is a signature for charged Higgs bosons. A measurement of event yield ratios $R_{l}$ for $t \bar{t} \rightarrow b \bar{b}+l \tau_{\text {had }}+N v$ and $t \bar{t} \rightarrow b \bar{b} l l^{\prime}+N v$, where $N v$ stands for any number of neutrinos and where $l$ and $l^{\prime}$ are electrons and muons, with $l \neq l^{\prime}$, was presented:

$$
R_{l}=\frac{B\left(t \bar{t} \rightarrow b \bar{b}+l \tau_{\mathrm{had}}+N v\right)}{B\left(t \bar{t} \rightarrow b \bar{b} l l^{\prime}+N v\right)}
$$

This analysis used events passing a single-lepton trigger with an $E_{\mathrm{T}}$ threshold of $20 \mathrm{GeV}$ or 22 $\mathrm{GeV}$ for electrons and a $p_{\mathrm{T}}$ threshold of $18 \mathrm{GeV}$ for muons. In order to select a sample enriched in $t \bar{t}$ events, the following selection was used: one charged lepton $l(e, \mu)$ having $E_{\mathrm{T}}>25 \mathrm{GeV}(e)$ or $p_{\mathrm{T}}>25 \mathrm{GeV}(\mu)$ and matched to the corresponding trigger object; at least two jets having $p_{\mathrm{T}}>20$ $\mathrm{GeV}$ and $|\eta|<2.4$, including exactly two $b$-tags; either exactly one $\tau$ jet with $p_{\mathrm{T}}^{\tau}>25 \mathrm{GeV}$ and $|\eta|<2.3$ with no additional charged lepton, or exactly one additional charged lepton $l$ with $E_{\mathrm{T}}$ or $p_{\mathrm{T}}$ above $25 \mathrm{GeV}$ and a different flavour than the trigger-matched lepton; $E_{\mathrm{T}}^{\text {miss }}>40 \mathrm{GeV}$. The selected events are then classified into two categories according to the single-lepton trigger that they fire: an electron trigger (EL) or a muon trigger (MU). Each category contains $\tau_{\text {had }}+$ lepton and dilepton $\left(l l^{\prime}\right)$ events. The lepton appearing first in the final state is, by convention, matched to the corresponding trigger object. The EL category therefore consists of $e+\tau_{\text {had }}$ and $e+\mu$ events, while the MU category contains $\mu+\tau_{\text {had }}$ and $\mu+e$ events. A significant background consists of events with reconstructed electrons and muons arising from the semileptonic decay of hadrons with $b$ - or $c$-quarks, from the decay-in-flight of $\pi$ or $K$ mesons and, in the case of electrons, from $\pi^{0}$ mesons, photon conversions or shower fluctuations. These are referred to as misidentified leptons. Two data samples are defined, which differ only in the lepton identification criteria. The 
tight sample corresponds to the selection used in the analysis and contains mostly events with real leptons. The loose sample is obtained by loosening the isolation and identification requirements, and it contains mostly events with misidentified leptons. The efficiencies for a real or misidentified lepton, respectively, to be detected as a tight lepton, are determined from data. About $51 \%$ of the simulated $t \bar{t}$ events in the $\tau_{\text {had }}+$ lepton final state contain a $\tau$ jet matched to a hadronically decaying $\tau$ lepton at the generator level. In the other events, the $\tau$ jet is misidentified. Data-driven methods are used in order to determine the probability of misidentification from electrons and hadronic jets. The majority of misidentified $\tau$ jets in the final event selection originate from jets, for which the misidentification probability depends on the initial parton (light quark, heavy-flavour quark or gluon). All jet types occur in $t \bar{t}$ events, and it is not possible to accurately predict the fraction of each of them, potentially leading to a large systematic uncertainty on the jet $\rightarrow \tau_{\text {had }}$ misidentification probability. However, the influence of all jet types other than light-quark jets can effectively be eliminated by categorising all events in terms of the charge of the lepton relative to the $\tau$ jet as opposite- sign (OS) or same-sign (SS) events. All processes with gluon and $b$-quark jets produce positively and negatively charged misidentified $\tau$ objects at the same rate. On the other hand, the light-quark jet component in SS events represents both charge misreconstruction and quarks which fragment such that the leading charged particle does not have the same charge as the initial quark. Giving a negative weight to the SS events therefore cancels, on average, the gluon and heavy-flavour-quark jet contributions from the OS events, leaving only light-quark jets misidentified as $\tau$ jets. The rate at which light-quark jets are misidentified as $\tau$ candidates is derived using a region enriched with $W^{+} \rightarrow 2$ jets events in the data. The $W^{+} \rightarrow 2$ jets events are classified as OS and SS events using the charges of the lepton and the $\tau$ candidate. Figures $2(\mathrm{a}, \mathrm{b})$ show the $m_{\mathrm{T}}$ distribution for OS, SS and OS-SS events fulfilling the $W^{+} \rightarrow 2$ jets selection. This demonstrates the cancellation of heavy-flavour-quark and gluon contributions. The number of tracks associated to jets misidentified as $\tau$ candidates is found to be poorly modelled in simulation. In order to correct the $\tau$ candidate selection efficiencies in simulation, $\tau$ track multiplicity scale factors are derived using OS-SS events fulfilling the $W^{+} \rightarrow 2$ jets selection, and are then applied to all jets misidentified as $\tau$ candidates in the simulation. The probability for a light-quark jet to be misidentified as a $\tau$ jet is measured in the data. OS events are given a weight +1 and SS events are given a weight -1 , in both the numerator and denominator of the jet $\rightarrow \tau_{\text {had }}$ misidentification probability. After OS-SS subtraction, the selected events mostly contain $\tau$ candidates coming from light - quark jets and, to a much lesser extent, electrons, muons, and true hadronically decaying $\tau$ leptons.

For each of the four final states considered, the OS-SS event yield $N$ can be split into two contributions: from $t \bar{t}$ events and from all other SM processes except $t \bar{t} \rightarrow b \bar{b} W^{+} W^{-}$. The event yield ratios are defined as:

$$
R_{e}=\frac{N\left(e+\tau_{\mathrm{had}}\right)}{N(e+\mu)} \quad R_{\mu}=\frac{N\left(\mu+\tau_{\mathrm{had}}\right)}{N(\mu+e)}
$$

Systematic uncertainties arise from the simulation of the electron and muon triggers, from the reconstruction and identification efficiencies of the physics objects, as well as from the energy/momentum scale and resolution for these objects. The total systematic uncertainty is around $10 \%$ for both ratios $R_{e}$ and $R_{\mu}$, where the largest uncertainties come from the $\tau$-ID efficiency, the $t \bar{t}$ generator and 


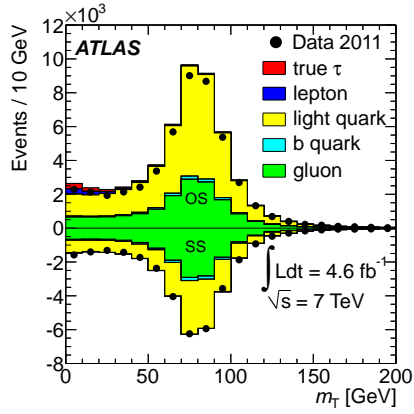

(a)

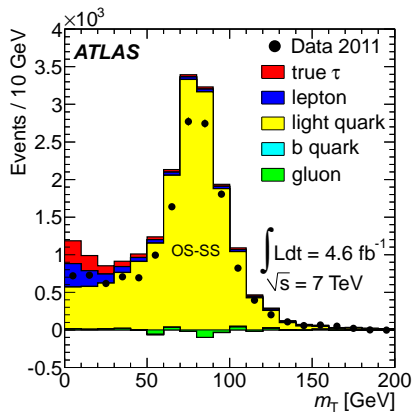

(b)

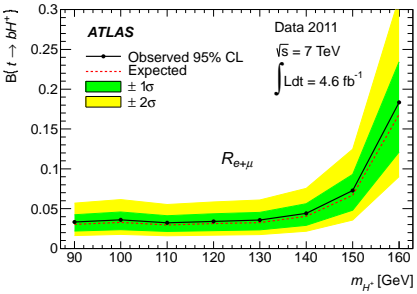

(c)

Figure 2: (a,b) Distributions of the transverse mass $m_{\mathrm{T}}$ for events fulfilling the $W^{+} \rightarrow 2$ jets selection. The SS events are subtracted from the OS events. (c) Upper limits on $B\left(t \rightarrow b H^{+}\right)$derived from the event yield ratio $R_{(e+\mu)}$, as a function of the charged Higgs boson mass, obtained with the assumption $B\left(H^{+} \rightarrow \tau v\right)=1$. The solid line in the figure is used to denote the observed 95\% CL upper limits, while the dashed line represents the expected exclusion limits. The green and yellow regions show the $1 \sigma$ and $2 \sigma$ error bands [2].

parton shower variations, and backgrounds with misidentified leptons. To test the compatibility of the data with the background-only or the signal+background hypotheses, a profile likelihood ratio [13] is used with $R_{e}$ and $R_{\mu}$ as the discriminating variables. The systematic uncertainties are incorporated via nuisance parameters, and the one-sided profile likelihood ratio is used as a test statistic. No significant deviation from the SM prediction is observed in the data. Exclusion limits are set on the branching fraction $B\left(t \rightarrow b H^{+}\right)$by rejecting the signal hypothesis at the $95 \%$ CL [14]. They are first set for electron-triggered and muon-triggered events separately, and then using a global event yield ratio $R_{e+\mu}$. Using this global event yield ratio, upper limits in the range $3.2 \% 4.4 \%$ are obtained on $B\left(t \rightarrow b H^{+}\right)$for charged Higgs boson masses in the range $90-140$ $\mathrm{GeV}$, as shown in Figure 2(c).

\section{Multi-Higgs-boson cascade search}

This search looks for particles in an extension to the SM that includes heavier Higgs bosons in addition to a light neutral Higgs boson, $h^{0}$, with mass $m_{h^{0}}=125 \mathrm{GeV}$. Rather than assuming a particular theoretical model, this analysis follows a simplified model approach by searching for a specific multi-Higgs-boson cascade topology. New particles are searched for in the final state $W^{ \pm} W^{\mp} b \bar{b}$, via the process $g g \rightarrow H^{0}$ followed by the cascade, $H^{0} \rightarrow W^{\mp} H^{ \pm} \rightarrow W^{\mp} W^{ \pm} h^{0} \rightarrow$ $W^{ \pm} W^{\mp} b \bar{b}$. This final state also appears in top-quark pair production. In this search, one of the $W$ bosons is assumed to decay to hadrons leading to jets and the other one decays to an electron plus a neutrino ( $e+$ jets $)$ or a muon plus a neutrino $(\mu+$ jets). Boosted decision trees (BDTs) are used to distinguish the Higgs-boson cascade events from the predominantly $t \bar{t}$ background. Events are selected using single-lepton triggers with $p_{\mathrm{T}}$ thresholds of 24 or $36 \mathrm{GeV}$ for muons and 24 or 60 $\mathrm{GeV}$ for electrons (the lower momentum triggers also apply isolation requirements). Events are required to have exactly one reconstructed isolated electron or muon matching the corresponding trigger object and a primary vertex reconstructed from at least five tracks, each with $p_{\mathrm{T}}>400$ 
$\mathrm{MeV}$. At least four jets with $p_{\mathrm{T}}>25 \mathrm{GeV}$ and $|\eta|<2.5$ are required, of which at least two must be identified as b jets. Additionally, in the $e+$ jets channel $E_{\mathrm{T}}^{\text {miss }}>30 \mathrm{GeV}$ and $m_{\mathrm{T}}^{W}>30 \mathrm{GeV}$, while in the $\mu+$ jets channel $E_{\mathrm{T}}^{\text {miss }}>20 \mathrm{GeV}$ and $m_{\mathrm{T}}^{W}+E_{\mathrm{T}}^{\text {miss }}>60 \mathrm{GeV}$. The Higgs-boson cascade event reconstruction begins with identification of the leptonically decaying $W$ boson. It is assumed that the missing transverse momentum is due to the resulting neutrino. The neutrino pseudorapidity is set to the value which results in an invariant mass of the lepton and neutrino closest to the nominal $W$-boson mass. Next, the two $b$-tagged jets are used to reconstruct the lightest Higgs-boson candidate, $h^{0}$. The hadronically decaying $W$ boson is identified from the remaining jets as the pair with reconstructed dijet mass closest to the nominal $W$-boson mass. The charged Higgs-boson candidate $H^{+}$is constructed from the light $h^{0}$ and the $W$-boson candidate which gives the larger value of $m_{H^{+}}$. The heavy neutral Higgs-boson candidate $H^{0}$ is then formed as $b \bar{b} W W$. Figure 3 illustrates the reconstructed mass distributions for the $h^{0}, H^{+}$, and $H^{0}$ in simulation for selected mass values.

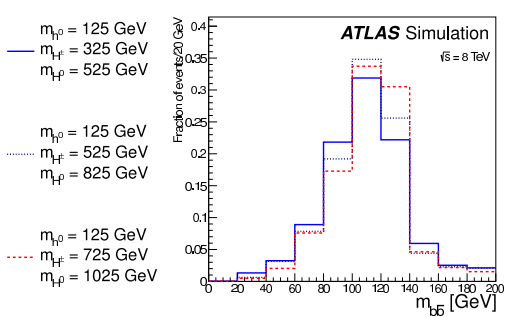

(a)

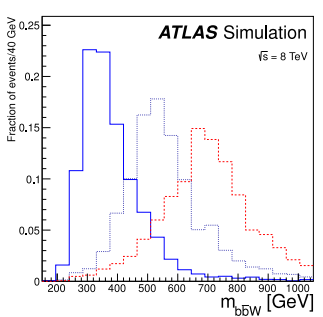

(b)

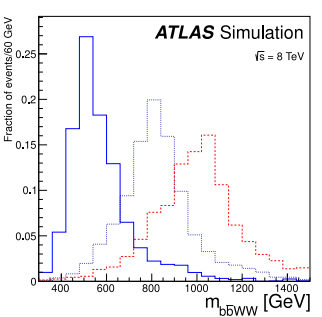

(c)

Figure 3: Distributions of reconstructed masses in simulation for the three Higgs bosons in the cascade; the lightest Higgs boson, $h^{0}$ (left, as $m_{b \bar{b}}$ ), the charged Higgs boson, $H^{ \pm}$(middle, as $m_{b \bar{b} W}$ ), and the heavy Higgs boson, $H^{0}$ (right, as $m_{b \bar{b} W W}$ ) shown for three example mass hypotheses [3].

A multivariate analysis is performed to distinguish the Higgs-boson cascade from $t \bar{t}$ events. Several reconstructed kinematic quantities, including the invariant masses of the Higgs-boson candidates as described above, are used as inputs to a BDT classifier, provided in the TMVA [15] package. TMVA provides a ranking for the input variables, which is derived by counting how often an input variable is used to split decision tree nodes, and by weighting each split occurrence by the square of the gain in signal-to-background separation it has achieved and by the number of events in that node. Seven kinematic variables are chosen to achieve the best expected result across the entire signal mass grid: $m_{b \bar{b}}, m_{b \bar{b} W}, m_{b \bar{b} W W}, \Delta R(b \bar{b})$ and the hadronic and leptonic masses of the top-quarks, and their difference. Since the kinematics of the Higgs-boson cascade vary greatly with the masses of the heavy and intermediate Higgs bosons, a different BDT is trained for each signal mass hypothesis. For each mass point, a final threshold is chosen for its respective BDT output which gives the best expected sensitivity, measured using the same confidence-level calculations as applied to the data. A counting experiment is then performed using events that pass those BDT output thresholds. In this way, the BDT thresholds divide the SPR into nonorthogonal signal regions, one for each signal mass point. The modelling of the SM backgrounds is validated in three background-dominated control regions. The control regions retain the requirements of one lepton and at least four jets, and each region has additional requirements. In control regions 
with fewer than two $b$-tagged jets, the two jets with the highest $b$-tagging scores are used to reconstruct the lightest Higgs boson, $h^{0}$. The following control regions are used:

- Control Region 1 (CR1): at least four jets, exactly one lepton and no $b$-tagged jets. Validates primarily the $W+$ jets modelling.

- Control Region 2 (CR2): at least four jets, exactly one lepton and exactly one $b$-tagged jet. This region validates primarily the modelling of the $t \bar{t}$ background.

- Control Region 3 (CR3): at least four jets, exactly one lepton, at least two $b$-tagged jets, and $m_{b \bar{b}}>150 \mathrm{GeV}$. This region focuses primarily on validation of the modelling of the $t \bar{t}$ background with kinematics similar to the hypothetical signal, but is background enriched due to the $m_{b \bar{b}}>$ requirement.

Figure $\uparrow$ illustrates the modelling of the Higgs-boson mass reconstruction in CR1, CR2 and CR3. The data and simulation agree within total uncertainties over the entire phase space. In addition, the BDT output in each of the three control regions is compared to the predicted output and found to agree within statistical and systematic uncertainties. The observed yields are found to be consistent with SM background expectations, within uncertainties. The BDT outputs for three example signal mass points are illustrated in Figure 5 .

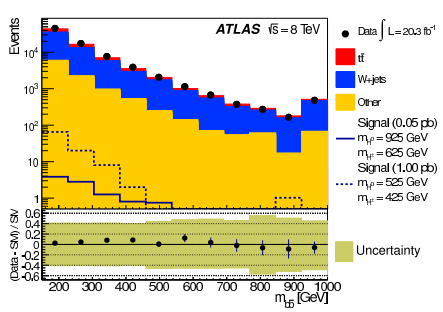

(a)

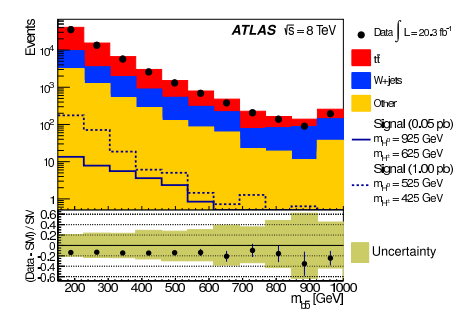

(b)

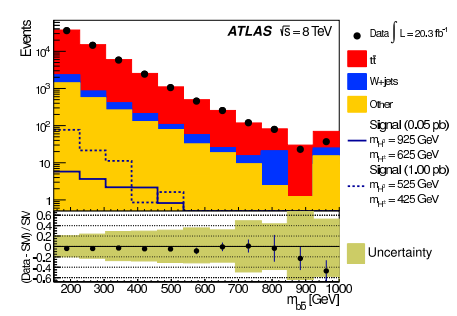

(c)

Figure 4: Distributions of $m_{b \bar{b}}$ with uncertainties in the control regions (a) CR1, (b) CR2 and (c) CR3. The data (black points) are compared to the background model (stacked histogram) [3].

The 95\% CL production cross - section upper limits for the various signal hypotheses are obtained, with the profile likelihood ratio of the number of events that pass the BDT threshold as the test statistic [13]. Systematic uncertainties are treated as nuisance parameters and the calculation uses the asymptotic approximation [13]. Since the signal regions are correlated, background-only pseudoexperiments are used to estimate the expected distribution of the $p$ values in all the signal regions, accounting for the correlations. The observed distribution of $p$ values is found to be consistent with the expectation from pseudoexperiments. The expected and observed limits as a function of the $\mathrm{H}^{0}$ and $\mathrm{H}^{+}$masses are illustrated in Figure 6. The limits are the weakest in low Higgs-boson mass regions due to the poorer separation between $t \bar{t}$ and signal events. The observed cross-section limits are compared to the predictions for a heavy Higgs boson with SM-like $g g$-fusion production (Figure 6). The theoretical production cross section of a heavy SM-like Higgs boson (only gluon fusion is considered) is calculated in the complex-pole scheme using the dFG [16] program, 


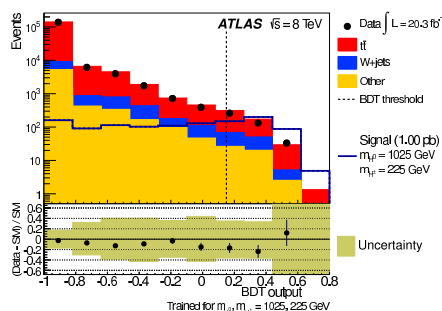

(a)

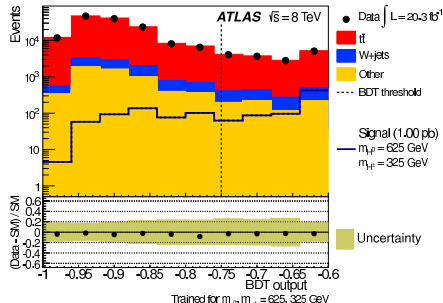

(b)

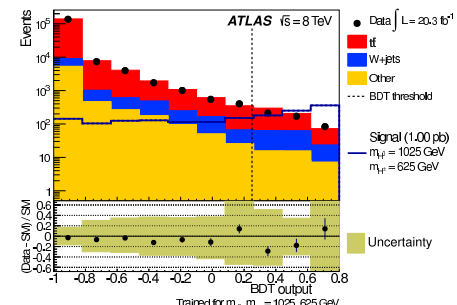

(c)

Figure 5: Distributions of the BDT output in the signal regions for three example signal mass points, (a) $m_{H^{0}}, m_{H^{ \pm}}=1025,225 \mathrm{GeV}$, (b) $m_{H^{0}}, m_{H^{ \pm}}=625,325 \mathrm{GeV}$, and (c) $m_{H^{0}}, m_{H^{ \pm}}=1025,625 \mathrm{GeV}$. Signal histograms have been scaled to a production cross section of $1 \mathrm{pb}$. BDT thresholds are shown as dashed lines for each mass point. The background model is shown as the coloured stacked histogram [3].

to NNLO in QCD. NLO electroweak corrections are also applied, as well as QCD soft-gluon resummations up to next-to-next-to-leading log. Using this benchmark, the cross-section upper limits observed are greater than the theoretical cross sections of the heavy Higgs boson, $H^{0}$, for all mass points tested. Therefore, the current limits are not stringent enough to exclude models with SM-like production rates even with $100 \%$ branching ratios for both $H^{0} \rightarrow H^{+} W^{-}$and $H^{+} \rightarrow h^{0} W^{+}$and SM values for $B\left(h^{0} \rightarrow b \bar{b}\right)$. The limits are most stringent in the high $H^{0}$ and $H^{+}$ mass regions, where the ratio of the limits to the theoretical cross section is nearly unity.

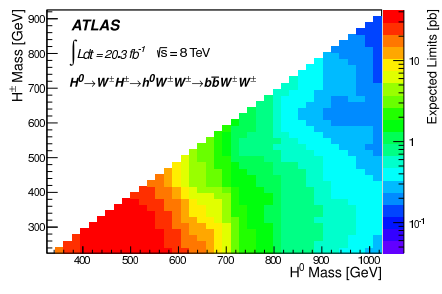

(a)

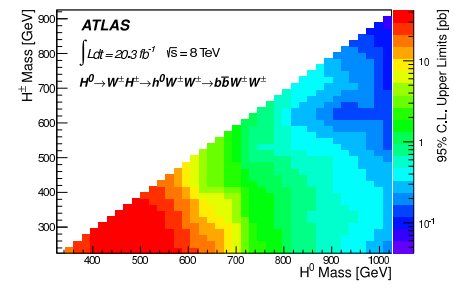

(b)

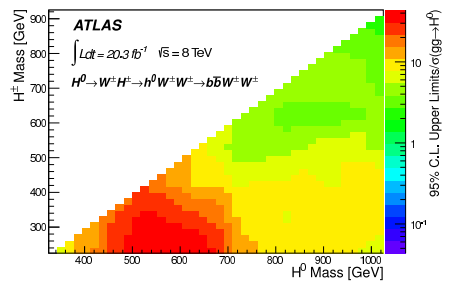

(c)

Figure 6: The expected (a) and observed (b) 95\% C.L. upper limits on the cross section for $g g \rightarrow H^{0} \rightarrow$ $W H^{ \pm} \rightarrow W W h \rightarrow W W b b$ as a function of $m_{H^{0}}$ and $m_{H^{ \pm}}$. The ratio (c) of the observed $95 \%$ C.L. upper limits on the cross section to the theoretical cross section for a heavy Higgs boson produced via gluon-gluon fusion at the SM rate [3].

\section{Conclusions}

Searches for charged Higgs bosons with the ATLAS detector [1-3] are presented. Using a dataset corresponding to an integrated luminosity of $4.7 \mathrm{fb}^{-1}$ recorded by the ATLAS detector in proton-proton collisions at a centre-of-mass energy of $\sqrt{s}=7 \mathrm{TeV}, 95 \%$ confidence level (CL) upper limits on $B\left(t \rightarrow b H^{+}\right)$varying between $5 \%$ and $1 \%$ for $H^{+}$masses between $90 \mathrm{GeV}$ and $150 \mathrm{GeV}$, assuming $B\left(H^{+} \rightarrow c \bar{s}\right)=100 \%$ are obtained, as well as upper limits in the range $3.2 \%$ to 
4.4\% on the branching fraction $B\left(t \rightarrow b H^{+}\right)$for charged Higgs boson masses in the range $90-140$ $\mathrm{GeV}$ (assuming $\left.B\left(H^{+} \rightarrow \tau v\right)=100 \%\right)$. Finally, using a dataset corresponding to $20.3 \mathrm{fb}^{-1}$ recorded by the ATLAS detector in proton-proton collisions at a centre-of-mass energy of $\sqrt{s}=8 \mathrm{TeV}$, upper limits on the cross section to the theoretical cross section for a heavy Higgs boson produced via gluon-gluon fusion at the SM rate are obtained, in a search for a multi-Higgs-boson cascade topology. Those limits range from 0.065 to $43 \mathrm{pb}$ as a function of $H^{0}$ and $H^{ \pm}$masses, with $m_{h^{0}}$ fixed at $125 \mathrm{GeV}$. No deviation from the SM predictions is observed.

\section{References}

[1] ATLAS collaboration, Eur.Phys.J.C, 736 (2013) 2465

[2] ATLAS collaboration, JHEP 1303 (2013) 076 (2013)

[3] ATLAS collaboration, Phys. Rev. D 89, 032002 (2014)

[4] ATLAS Collaboration, Phys. Lett. B 716(1), 1 (2012)

[5] CMS Collaboration, Phys. Lett. B 716(1), 30 (2012)

[6] Y. Grossman, Nucl. Phys. B 426, 355 (1994)

[7] S. Dimopoulos, H. Georgi, Nucl. Phys. B 193, 150 (1981)

[8] S. Heinemeyer et al., Comput. Phys. Commun. 124, 76 (2000)

[9] ATLAS collaboration, 2008 JINST 3 S08003

[10] T. Aaltonen et al. (CDF Collaboration), Phys. Rev. Lett. 103, 101803 (2009)

[11] ATLAS Collaboration, Phys. Lett. B 717, 330 (2012)

[12] ATLAS Collaboration, Eur. Phys. J. C 72, 2039 (2012)

[13] G. Cowan et al., Eur. Phys. J. C 71, 1554 (2011)

[14] A.L. Read, J. Phys. G 28 (2002) 2693

[15] A. Hocker et al., Proc. Sci., ACAT (2007) 040

[16] S. Heinemeyer et al. (LHC Higgs Cross Section Working Group), arXiv:1307.1347, CERN-2013-004 